\title{
OLD AND NEW METHODS OF OBSERVING POLAR MOTION
}

\author{
R. O. VICENTE \\ Dept. of Applied Mathematics, Faculty of Sciences, Lisbon University, Portugal
}

\begin{abstract}
The motion of the pole has been observed by means of visual telescopes, employed by the ILS, photographic zenith tubes and astrolabes. It is pointed out the difficulties of combining the results of different types of instruments employing several observation techniques.

The new astronomical methods of Doppler observations on satellites and laser pulses sent to the Moon give another possibility of determining polar motion with greater precision. It is forecast that the results will not agree with the older ones and, in order to compare the methods, it is suggested that stations should be equipped with both techniques of detecting polar motion.
\end{abstract}

The motion of the pole was first detected by observations of latitude employing visual instruments. The importance of the results obtained led to the setting up of the International Latitude Service (ILS), composed of a certain number of stations, equipped with the same type of visual instruments and forming a chain situated in the same parallel $\left(39^{\circ} 8^{\prime} \mathrm{N}\right)$. This observing technique was the most accurate at the beginning of this century when the service began to operate.

Several investigations of the precision obtained in these observations show that the mean error of a single determination of latitude is about 0"17 (Nicolini and Fichera, 1970), considering the different stations of the ILS.

Research done in the construction of instruments, permitting the study of the motion of the pole, led to photographic zenith tubes and astrolabes. The precision obtained with a photographic zenith tube corresponds to a standard deviation of 0.083 for one night of observation. The researches of Débarbat (1969) show that the observations made with astrolabes can have the same precision as the photographic zenith tube.

We have these different types of instruments employing observation techniques and methods of reduction of the observations that are distinct, and leading, therefore, to different accuracies.

The chain formed by the International Polar Motion Service (IPMS) instruments has the great advantage of being composed the same type of instruments and also of employing the same programme of observations. The results obtained from this chain of instruments have led to the possibility of drawing the curves of polar motion that have been published by the several directors of this international service.

However, more recently the Bureau International de l'Heure (BIH), under Guinot (for instance, 1968), has published data concerning the motion of the pole, employing the observations made with the types of instruments above mentioned and located at different latitudes. The curves of polar motion deduced from these data, obtained from a chain of instruments distinct from the IPMS chain, do not agree with the results of the IPMS. 
This work has been very useful in showing the anomalies that appear in the researches on polar motion, based on data obtained by instruments that have precisions of the same order of magnitude. We can say that these instruments and observation techniques give us a precision that corresponds to the determination of the coordinates of a place on the Earth with an error of the order of magnitude of $1.2 \mathrm{~m}$ (Vicente, 1968).

A very important contribution to this absurd state of things is due to the fact that the published results of the ILS and IPMS show such a lack of homogeneity, as has been pointed out by Vicente and Yumi (1969), in spite of the great advantages of using the same type of instruments and observing programmes. This example gives us a measure of the difficulties of combining different types of instruments employing varied programmes of observation.

We have now to consider the observations about polar motion furnished by the new astronomical methods of Doppler observations on satellites and laser pulses reflected by retro-reflectors situated on the Moon or on artificial satellites.

The Doppler observations employing artificial satellites permit the determination of the pole coordinates with a precision of one metre (Anderle and Beuglass, 1970). The utilisation of different satellites does not affect the precision obtained but the number and position of the observing stations alter the precision of the results. We can, therefore, conclude that this new astronomical technique gives, at the moment, a precision of the same order of magnitude as that of the older astronomical methods, but there is the great advantage that the pole coordinates are determined by a quite different type of observations. There are, however, certain difficulties peculiar to the Doppler observations such as, for instance, the effects of the gravity field of the Earth on the satellites.

The comparison of Doppler results with the results obtained by the older methods shows discrepancies of the order of $1 \mathrm{~m}$. We have to remember that the location of the observing stations is quite different, and this is certainly one of the sources that contribute to the difficulties of comparing the results.

The other new method of astronomical observation of polar motion is based on laser pulses sent by optical telescopes and reflected by retro-reflectors placed on the Moon. This method needs large telescopes and the best type of lasers, but further research will make it possible to employ telescopes of moderate size. For these reasons, the number of observing stations is restricted at the present time. The precision obtained is of the order of $0.3 \mathrm{~m}$ with the possibility of further improvement (Alley et al., 1970). There are also laser experiments employing artificial satellites.

The comparison of the laser methods with the other methods shows that we can attain a precision of an order of magnitude greater than the precision reached by the above mentioned techniques. This fact shows that we can forecast that the curve of polar motion which can possibly be obtained in the near future from laser methods will not agree with the existing ones.

The possibility of obtaining such greater accuracies implies that we cannot ignore any more the geophysical phenomena associated with the location of the stations such 
as, for instance, Earth tides, to mention only one of the better known local motions of the observing stations.

We have already the example of different curves of polar motion deduced from instruments of comparable precision and, therefore, we are going to have more difficulties in comparing observations deduced from quite new methods. This difficulty is, in another way, a great advantage because we shall be able to have the possibility of checking polar motion by completely different astronomical techniques.

The International Latitude Observatory of Mizusawa offers an excellent example of the advantages of having several instruments situated side by side, employing the old methods of studying polar motion.

Considering the difficulties mentioned and the forecast of greater problems in the comparison of the results obtained by the old and new methods of observing polar motion, it is suggested that some of the existing stations, employing either one or the other of the methods described, should be so equipped that they could use methods different from the one they have, in particular the new astronomical methods.

\section{References}

Alley, C. O., Chang, R. F., Currie, D. G., Poultney, S. K., Bender, P. L., Dicke, R. H., Wilkinson, D. T., Faller, J. E., Kaula, W. M., MacDonald, G. J. F., Mulholland, J. D., Plotkin, H. H., Carrion, W., and Wampler, E. J.: 1970, Science 167, 458.

Anderle, R. J. and Beuglass, L. K.: 1970, Bull. Géodés., No. 96.

Débarbat, S.: 1969, Astron. Astrophys. 1, 334.

Guinot, B.: 1968, Annuaire Bureau Longitudes, p. 290, Gauthier-Villars.

Nicolini, T. and Fichera, E.: 1970, Risultati del Servizio Internazionale delle Latitudini X.

Vicente, R. O.: 1968, Geophys. J. 14, 475.

Vicente, R. O. and Yumi, S.: 1969, Publ. Int. Lat. Obs. Mizusawa 7, No. 1.

\section{DISCUSSION}

E. P. Fedorov: If we consider the results of latitude observation as a stochastic process, we should study the spectrum of the errors. In this case the comparison is not in favour of the PZT's.

$R$. O. Vicente: But the order of magnitude of the errors is about the same, and that is the point of view we are concerned with in this paper.

E. P. Fedorov: Do you believe that the single value, namely the mean error of one observation is sufficient for estimation of the precision of latitude observation?

$R$. O. Vicente: No, the value presented is only to give an idea of the order of magnitude expected.

$H$. Jeffreys: How big are the differences between ILS and Guinot?

Answer (by B. Guinot): To question of Jeffreys. The differences between IPMS and BIH results are mainly annual and they are due to both the IPMS and the BIH because of the existence of local annual errors due to refraction and not only to the catalogues of stars. Further comments will be given with Paper 2.4 . 